\title{
BALANCE IMPROVEMENTS AND GOLF SWING KINEMATIC CONSISTENCY WITH EIGHT WEEK BALANCE INTERVENTION
}

\author{
(iD) Meredith K. Owen ${ }^{1}$ \\ Julia A. Gambill ${ }^{2}$ \\ Katerina N. Van \\ Damme $^{3}$ \\ Jordan E. Byrd ${ }^{4}$ \\ John D. \\ DesJardins $^{5+}$
}

\author{
${ }^{1,2, s, 5}$ Department of Bioengineering, Clemson University, Clemson, USA. \\ 'Email:mko@clemson.edu Tel:864-508-6571 \\ 'Email:jagambi@clemson.edu Tel: 803-727-9687 \\ ${ }^{3}$ Email:kvandam@,clemson.edu Tel: 202-246-7706 \\ ${ }^{5}$ Email:jdesjar@clemson.eduTel:864-650-0748 \\ ${ }^{4}$ Department of Athletics, Clemson University, Clemson, USA. \\ ‘Email:jordanb@clemson.edu Tel: 864-637-801s
}

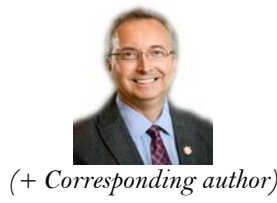

ABSTRACT

The purpose of the study was to isolate the relationship between balance and swing kinematics for collegiate level male golfers. Eight National Collegiate Athletic Association (NCAA) Division I golfers completed an eight-week training program targeting improving balance. All golfers were assessed for postural stability and swing kinematics before and after the balance training program. Golfers completed 10 swings with a driver, and swing kinematics were measured using $3 \mathrm{D}$ motion capture (Qualisys $\mathrm{AB})$. Postural stability results showed decreases in center-of-pressure (COP) deviation for single leg, eyes closed stance conditions were significant $(\mathrm{p}<0.05)$. Changes in Balance Error Scores (BES) were not significant $(\mathrm{p}>0.05)$ for any stance condition following training intervention. Range values (maximum-minimum) for club head speed (CHS), club path, and face angle stayed the same or decreased following the training intervention but were not significant $(\mathrm{p}>0.05)$. Key positions in the golf swing, including pelvis and torso angles at the top of the swing and impact were unaffected by the balance training $(\mathrm{p}>0.05)$. Pearson's values indicated a low degree of correlation between postural stability and swing kinematic consistency, as measured by variance. While postural stability improved following golf specific balance training, individual swing kinematics and swing kinematics consistency were unaffected.

Contribution/Originality: This study is one of very few studies which have investigated consistency in golf swing kinematics as well as the effect of strength and conditioning training, specifically balance training, on the consistency of golf swing kinematics.

\section{INTRODUCTION}

Although often considered a "leisure sport", golf requires extreme coordination, skill, and strength, especially to attain elite levels of performance. As more golfers aspire to compete at the highest levels, athletes and coaches are constantly seeking information on training methods that will improve golf skills. These programs must ultimately contribute to the overall goal of the sport, which is achieve the fewest strokes possible.

For golfers of all skill levels and age groups, strength, flexibility, and balance have been studied for direct effect on performance, and skilled golfers are likely to be more proficient in all three areas than their beginner counterparts (Sell, Tsai, Smoliga, Myers, \& Lephart, 2007). Improvements in strength, flexibility, and balance have been linked to increased club head speed (CHS) and increased driving distance (Doan, Newton, Kwon, \& Kraemer, 
2006; Fletcher \& Hartwell, 2004; Lephart, Smoliga, Myer, Sell, \& Tsai, 2007; Oranchuk, Mannerberg, Robinson, \& Nelson, 2020; Parker, Lagerhem, Hellström, \& Olsson, 2017). Strength and power have been targeted for improvement by several investigators employing a variety of techniques including free weights (Fletcher \& Hartwell, 2004), plyometrics (Doan et al., 2006; Fletcher \& Hartwell, 2004), core strengthening (Weston, Coleman, \& Spears, 2013) and isokinetic training (Parker et al., 2017). These methods have been found to improve selected strength measures as well as improve certain club and swing parameters such as CHS, driving distance, and ball speed. Flexibility has also been evaluated for its effect on golf performance. Such studies have evaluated the relationship between flexibility and CHS with (Doan et al., 2006) and without (Gordon, Moir, Davis, Witmer, \& Cummings, 2009; Marshall \& Llewellyn, 2017) an intervention. Doan et al. investigated the outcomes of an 11 week strength, power, and flexibility training and noted increases in flexibility and CHS without any negative effects on putting distance control (Doan et al., 2006). However, flexibility training was not isolated so direct effects of flexibility increases on CHS are difficult to determine. Furthermore, Gordon et al. found no significant correlation between rotational trunk flexibility and CHS for male golfers (Gordon et al., 2009).

Many of these studies utilize indirect measures of the golf swing, such as club head speed, ball speed, and carry distance, to determine the effects of the different training programs or physical traits being assessed. While these measures are important, the implementation of 3D motion capture allows for direct measures of golfer performance, such as body segment position and body segment velocity. Often, studies have utilized motion capture to determine kinematic differences between unskilled golfers and skilled or professional golfers (Cheetham, Martin, Mottram, \& Laurent, 2001; Cheetham et al., 2008; Sell et al., 2007), with skilled and professional golfers exhibiting significantly different biomechanical movements than their unskilled counterparts. Additionally, motion capture has allowed for direct comparison of the effects of different training methods on golfer kinematics. Using these methods, isokinetic strength training has been shown to have a slight effect on lead arm speed and acceleration (Parker et al., 2017).

While increases in driving distance and clubhead speed are often the metrics used to quantify the effectiveness of a certain training regimen, swing consistency should also be considered. A golfer's ability to consistently repeat the same golf swing could be hypothesized to be just as important as their ability to generate increased clubhead speed or greater carry distance for isolated swings. Previous researchers have indicated the importance of repetitively achieving the same swing movements and have taken note of the large number of variables that may affect a golfers ability to consistently repeat the same movements (Langdown, Bridge, \& Li, 2012). The concept of movement variability, introduced by Langdown et al. (2012) relates to the shot variability based on the task at hand, and is based on the movements of the golfer (Langdown et al., 2012). Small changes in the movements of the golfer can greatly affect the end result of the shot, with even a few degrees of clubhead movement resulting in a wide variety of shot variations. While this variability can be beneficial when attempting to perform a certain specialty shot, too much can have a negative effect on performance causing errant and unwanted shots. To date, there have been few studies analyzing variability in the golf swing. Weston et al. evaluated variability of several swing parameters following an 8 week core training program and found small improvements in the consistency of repeated CHS and ball backspin (Weston et al., 2013). However, variability in the movements of the golfer have been examined less frequently.

Strength, flexibility, and balance are well known contributors to golf performance. While these attributes have been examined together, and strength and flexibility have been studied in isolation, balance has yet to be investigated independently. To date, few studies have examined the impact of balance specific training on golf performance and even fewer have examined its effect on golf swing consistency. Balance is crucial for maintain consistent positioning throughout the golf swing. Golfers who have decreased single leg balance are more likely to lose posture, extend their hips early, and slide during the swing which affect the variability of each swing (Gulgin, Schulte, \& Crawley, 2014). Gulgin et al. (2014) indicate that for right-handed golfers, the inability to balance on the left side during weight shift to the lead leg, which can be isolated and determined using static balance assessments, can disrupt the golf swing leading to increased swing variability (Gulgin et al., 2014). Consistency, or reduced 
variability in the golf swing, is considered by coaches and players to be a contributor to improved performance. The goal of the present study was to examine the effects of an eight-week golf specific balance training protocol on postural stability improvements and golf swing kinematic consistency in intercollegiate golfers. It is hypothesized that a greater level of postural stability and improvements in postural stability would correlate to greater swing consistency and improvements in swing consistency, respectively.

\section{METHODS}

\subsection{Participants}

Following Institutional Review Board approval, eight NCAA division I golf athletes were recruited to participate in the study. All participants were male between the ages of 18 and 22 (age: $19.6 \pm 0.9$ years; weight: $79.2 \pm 12.9 \mathrm{~kg}$; height: $184.5 \pm 8.8 \mathrm{~cm}$ ). Most of the subjects did not maintain an active USGA handicap. Using an average of competitive rounds, the cohort score average was determined to be approximately 70 . All but one of the participants were right handed. Prior to completing any study related activities, subjects were informed of study related risks and voluntarily signed an informed consent form.

\subsection{Balance Intervention Training Protocol}

An eight-week golf specific balance training protocol was developed by the study investigators and the university strength and conditioning coach. The balance training protocol included four progressions designed to improve overall balance as it relates to golf specific movements. Table 1 lists the specific exercises and progressions completed during the eight-week study. Exercises target single leg training which has noted specific benefits for golfers including improving lateral stability and training in multiple planes in addition to improving balance according to the Titleist Performance Institute (TPI). TPI level 1 movement screening assesses single leg balance, and Gulgin et al. (2014) found this to be one of the most common physical limitations for golfers (Gulgin et al., 2014). By targeting specific single leg and lower body movements, the training protocol was designed to address balance and single leg balance weaknesses.

Table-1. 8-week balance training protocol.

\begin{tabular}{|c|c|c|c|}
\hline Exercises & Week & Sets & Reps (First Week/Second Week) \\
\hline \multicolumn{4}{|l|}{ Progression \#1 } \\
\hline \multirow{2}{*}{ Core Engaged SL Balance } & $1-2$ & 3 & $20 \mathrm{~s} / 30 \mathrm{~s}$ \\
\hline & $3-4$ & 3 & $30 \mathrm{~s}^{* *} / 30 \mathrm{~s}^{\wedge \wedge}$ \\
\hline \multirow[t]{2}{*}{ SL Balance } & $5-6$ & 3 & $30 \mathrm{~s}^{*} / 30 \mathrm{~s} * *$ \\
\hline & $7-8$ & 3 & $30 \mathrm{~s}^{\wedge} / 30 \mathrm{~s}^{\wedge \wedge}$ \\
\hline \multicolumn{4}{|l|}{ Progression \#2 } \\
\hline Supported SL RDL & $1-2$ & 3 & 3 each leg/4 each leg (w/ 5 s hold) \\
\hline SL RDL and Reach & $3-4$ & 3 & 3 each leg/4 each leg (w/ 5 s hold) \\
\hline \multirow{2}{*}{ SL RDL to SL Balance } & $5-6$ & 3 & 4 each leg/5 each leg \\
\hline & $7-8$ & 3 & 3 each leg/4 each leg (Eyes-Closed) \\
\hline \multicolumn{4}{|l|}{ Progression \#3 } \\
\hline Crow Hop & $1-2$ & 3 & 3 each leg/4 each leg \\
\hline Hieden (stick landing) & $3-4$ & 3 & 3 each leg/4 each leg \\
\hline Reactive Hieden & $5-6$ & 3 & 3 each leg/4 each leg \\
\hline SL Barrier Hop & $7-8$ & 3 & 3 each leg/4 each leg \\
\hline \multicolumn{4}{|l|}{ Progression \#4 } \\
\hline SL Quarter Squat & $1-2$ & 3 & $20 \mathrm{~s} / 30 \mathrm{~s}$ \\
\hline \multirow{3}{*}{ SL Quarter Squat on Airex Pad } & $3-4$ & 3 & $20 \mathrm{~s} / 30 \mathrm{~s}$ \\
\hline & $5-6$ & 3 & $30 \mathrm{~s}^{*} / 30 \mathrm{~s}^{* *}$ \\
\hline & $6-7$ & 3 & $30 \mathrm{~s}^{\wedge} / \wedge \wedge$ \\
\hline
\end{tabular}


Participants were instructed on how to complete the exercises included in the balance training protocol and were asked to complete the exercises three times a week for eight weeks. The start of the balance training coincided with the start of the competitive collegiate season. Due to competitive travel schedules not all balance exercises were completed during standard strength and conditioning training sessions, but individuals were instructed to complete the exercises on their own when traveling.

\subsection{Testing Procedures}

\subsubsection{Postural Stability Assessment}

Postural stability was assessed for each subject following methods modified from those proposed by Goldie and colleagues (Goldie, Bach, \& Evans, 1989). Subjects were assessed at week o, prior to the start of the balance training, and at week 8 , the conclusion of the balance training. Subjects completed a series of trials targeting single leg and double leg balance ability. While standing on a multi-axis force plate (Accusway, AMTI, Watertown, MA), subjects were instructed to stand with either double leg support or single leg support and either an eyes open or eyes closed condition Table 2. Each stance was held for 15 seconds. Three trials of each stance were performed. For double-leg support stance conditions, subjects were instructed to stand with feet approximately shoulder-width apart and with weight evenly distributed. For single-leg support stance conditions, subjects were instructed to place the support foot at the center of the force plate and keep the raised leg at approximately 45 degrees. Base of support locations were marked and used for reference for the post-training assessment. During eyes-closed trials, subjects were directed to focus on a front facing target, assume the required stance position, and then close their eyes. Data collection began as soon as the subject's eyes were fully closed.

Additionally, each trial was assessed using a modified version of the Balance Error Scoring System (BESS) to score stance deviation errors (Bell, Guskiewicz, Clark, \& Padua, 2011). While the full BESS protocol was not completed for the study, the error scoring system developed for the BESS protocol was used to assign an error score for each stance condition. An investigator tallied errors during each stance condition trial. Such errors included opening eyes only during eyes closed stances, moving hands from hips, stepping, stumbling, or falling, abduction or flexion at the hip greater than 30 degrees, lifting heel or forefoot, and/or remaining out of instructed position for more than 5 seconds. All errors were weighted equally and were given a score of 1 point. If multiple errors occurred at the same time, it was recorded as only one error. This combination of BESS evaluation with objective postural stability measures captured by the force plate has been utilized by previous researchers (Riemann, Guskiewicz, \& Shields, 1999).

Table-2. Postural stability assessment order for testing.

\begin{tabular}{c|c|c}
\hline Trial & Stance Condition & Visual Condition \\
\hline 1 & Open Base, Double Leg support & Eyes Open \\
\hline 2 & Open Base, Double Leg support & Eyes Closed \\
\hline 3 & Right, Single Leg & Eyes Open \\
\hline 4 & Right, Single Leg & Eyes Closed \\
\hline 5 & Left, Single Leg & Eyes Open \\
\hline 6 & Left, Single Leg & Eyes Close \\
\hline
\end{tabular}

\subsubsection{Swing Kinematics Assessment}

Swing kinematics was measured using an 8-camera markered motion capture system (Qualisys AB, Gothenburg, Sweden). All swing trials were completed in an indoor/outdoor hitting bay with a turf mat. The hitting bay was comparable to typical hitting bays used for practice by the participants. Cameras were spaced equally around the perimeter of the hitting bay such that each reflective marker could be seen by a minimum of two cameras at every point during the golf swing. Figure 1 depicts the layout for the swing trials. All swings were captured at a frequency of $500 \mathrm{~Hz}$ and individual camera settings were tuned to provide optimal output in relation to location within the hitting bay. Prior to swing capture for each subject, calibration was completed following 
manufacturer recommended methods using a L-frame and calibration wand. Calibration was repeated until the calibration residual was less than $1.2 \mathrm{~mm}$, the manufacturer recommended setting for outdoor capture.

Prior to testing, subjects warmed-up following their own warm-up progression. However, all warm-up periods consisted of at least several practice shots with a 6 iron club and a driver, the two clubs used for testing. Subjects were outfitted with a total of 46 highly reflective, spherical markers with a diameter of $16 \mathrm{~mm}$ for dynamic testing. 42 markers were placed directly on the body and 4 markers were placed on the club. An additional 10 markers were included for a static calibration trial. Figure 2 shows the marker positions. Reflective markers were attached to the subject at specified anatomical locations and to the club using a skin-safe double sided adhesive. All locations, both on the body and the club, were specified for the golf specific data analysis Project Automation Framework (PAF) module developed by Qualisys (2019). Marker positions included locations on the head, shoulders, arms, torso, hips, legs, ankles, and shoes. For data collection, subjects completed a total of 15 swings, 5 swings with a 6 iron and then 10 swings with a driver. The iron swings were to familiarize the subjects with the test protocol and were not analyzed for this specific study. Each subject used their own clubs for all swings. Subjects were directed to aim at a common location for all shots and were instructed to use their normal hitting routine. A launch monitor (Trackman, Scottsdale, AZ, USA) was used to capture additional swing data including club head speed, club path, and face angle.

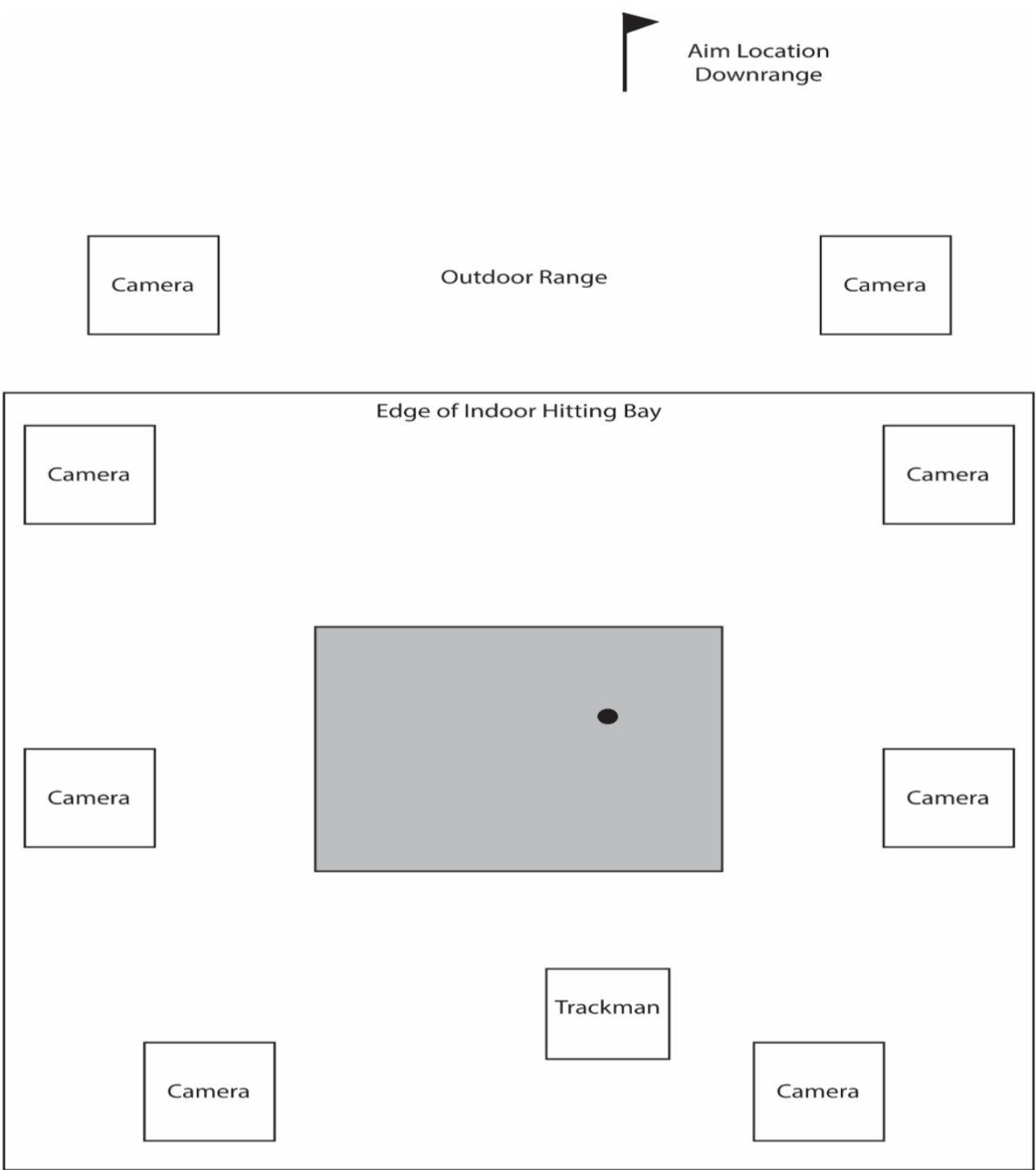

Figure-1. An indoor-outdoor hitting bay was used for all data collection. A turf mat was centered in the indoor bay and subjects were directed to hit shots at a location approximately 280 yards downrange. A Trackman launch monitor was placed in line with the golf ball behind the turf mat. 


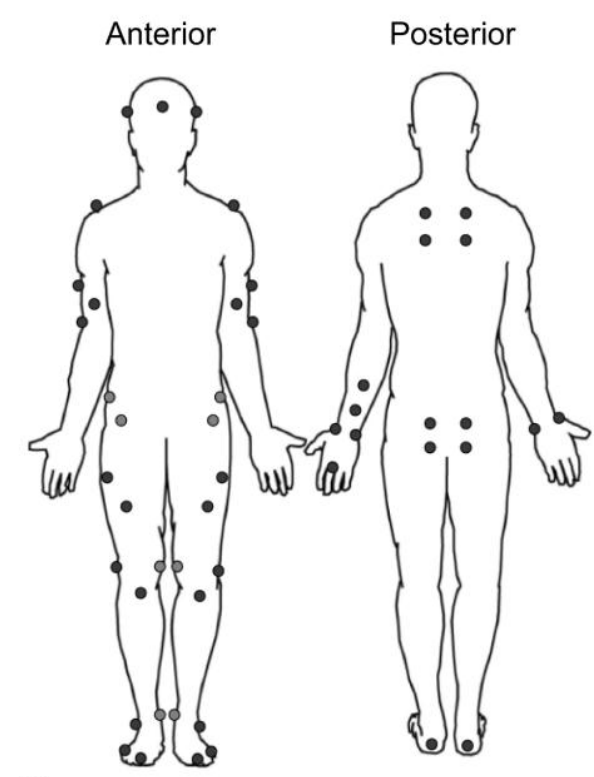

A)

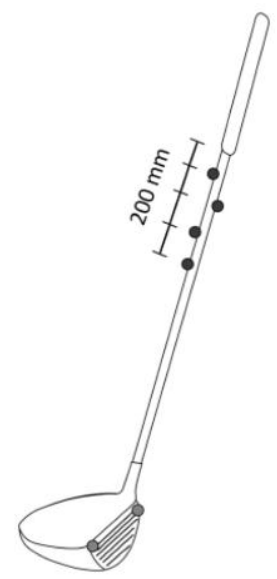

B)

Figure-2. Reflective markers (diameter $=16 \mathrm{~mm}$ ) were place on the A) upper and lower body and on B) the golf club. Locations shown in light grey depict makers used only for the stationary calibration trials.

\subsection{Data Analysis}

\subsubsection{Postural Stability}

All force plate data was analyzed using Balance Clinic software (for AMTI AccuSway Balance Platform, AMTI, Watertown, MA). Deviation in center-of-pressure (COP) was determined for each subject for each stance condition. COP deviation was defined as the area of the ellipse shape that encompasses $95 \%$ of the data points for data that is normally distributed. An average balance error score was determined for each stance condition.

\subsubsection{Swing Kinematics}

A total of 10 driver swings were collected for each participant. The top eight swings were further analyzed after data processing. 3D swing data were processed using Qualisys Track Manager (Qualisys AB, Gothenburg, Sweden) and Visual3D (C-Motion, Inc., Germantown, MD) enabled with the golf specific PAF module. 3D data, for all markers excluding those placed on the lead arm and club, were filtered with a bi-directional Butterworth lowpass filter with a cut-off frequency of $6 \mathrm{~Hz}$. For the lead arm and club, all data were filtered with a $10 \mathrm{~Hz}$ lowpass filter for the backswing, a $20 \mathrm{~Hz}$ lowpass filter for the downswing, and a $25 \mathrm{~Hz}$ lowpass filter for the follow through (Qualisys, 2019). All subjects completed the 8-week swing kinematic analysis; however, data for 1 subject could not be processed for week 8 due to a camera error during data collection and thus is not included. The data presented only includes the remaining 7 subjects. Only downswing kinematics were assessed as the goal of the swing is to reach impact with the greatest efficiency. Club head speed, face angle at impact, and club path were determined for the eight swings for each subject. Additionally, the pelvis and torso position were determined at the top, half down, and impact positions. The half down position was defined as lead arm parallel to the ground. The range for each set of values and the variance of the values were determined and used to estimate swing consistency.

\section{STATISTICAL ANALYSIS}

All data are reported as average \pm standard deviation unless stated otherwise. Balance error scores and COP data were analyzed using a paired $t$-test to determine if significant changes in postural stability occurred after the 8week balance training program. Range and variance values were determined for swing kinematic parameters. Preand post-training range values were also analyzed using a paired $t$-test. Consistency is defined as the variance in 
repeated swing measures. Statistical significance was defined as $\mathrm{p} \leq$ 0.05. Statistical analysis was performed using Excel (Microsoft) and JMP Pro (version 14.0; SAS Institute, Cary, NC).

\section{RESULTS}

\subsection{Postural Stability}

All subjects trended toward improved Balance Error scores (BES) following the 8-week balance training. BES improved significantly for single leg right stance with eyes close and for both eyes open and eyes closed single leg left stances Figure 3. The largest improvements were noted in the eye-closed conditions for both legs.

Changes in COP deviation were not significant for any stance position Figure 3. However, all stance conditions showed some improvement in COP deviation, indicated by reduced 95\% ellipse area, following the 8-week balance training. Greatest decreases in COP deviation were observed in the right single leg eyes closed and the left single leg eyes open stance conditions. All subjects showed improvements in COP deviation for at least one stance condition.

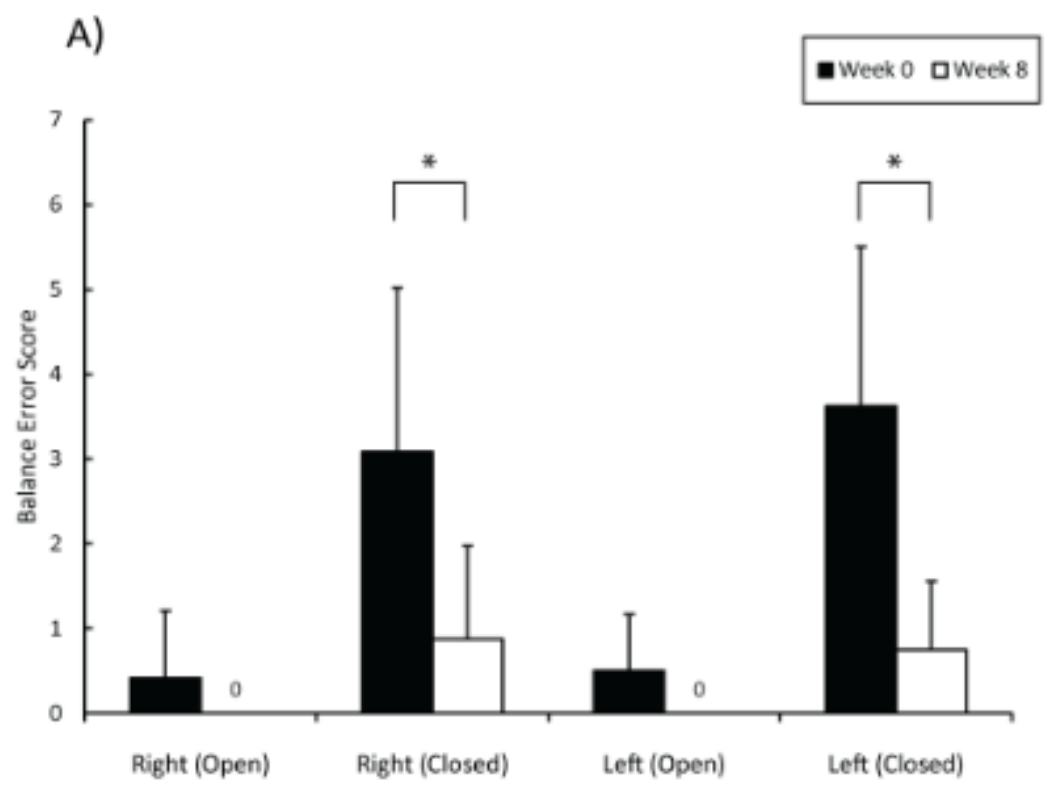

B)

week0 oweek 8

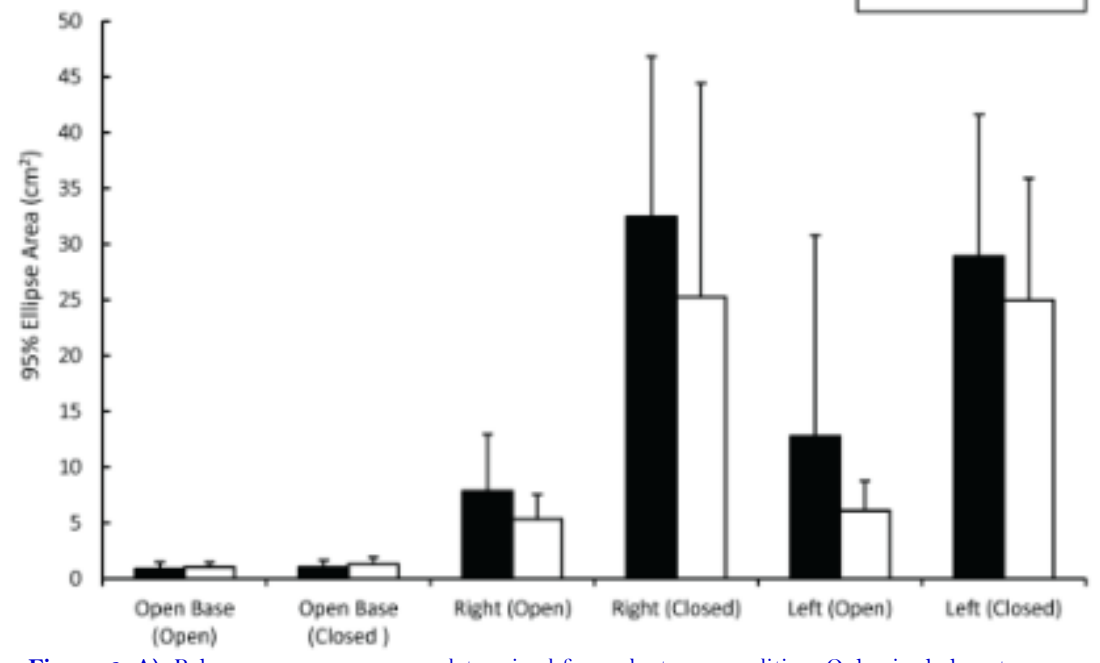

Figure-3. A). Balance error scores were determined for each stance condition. Only single leg stances had balance error scores greater than zero. Significant changes in BES were noted for both single leg stances with eyes closed condition. B) the $95 \%$ ellipse area of the COP deviation was determined for all stance conditions. No significant changes were found pre- to post-training. Note: ${ }^{*} \mathrm{p}<0.05$. 


\subsection{Swing Kinematics}

\subsubsection{Club Head Speed}

Club head speed neither improved nor was negatively impacted by the balance training. Differences in average CHS between pre- and post-balance training ranged from $-4.13 \mathrm{~km} / \mathrm{hr}$ to $4.38 \mathrm{~km} / \mathrm{hr}$ for individual subjects. CHS consistency was also unaffected by the balance training $(p=0.56)$. Average range for the cohort decreased only slightly Figure 4. CHS range difference spanned from an increase in $4 \mathrm{~km} / \mathrm{hr}$ to a decrease in $4 \mathrm{~km} / \mathrm{hr}$ for individual subjects. Variance changes spanned from a decrease in variance of 4.52 to an increase in variance of 5.43 for individual subjects.

\subsubsection{Face Angle and Club Path}

Club head face angle and club path were also unaffected by the balance training protocol (club path: $\mathrm{p}=1$, face angle: $\mathrm{p}=0.54$ ). The average range decreased slightly for face angle and stayed the same for club path Figure 4 . For individual subjects, differences in face angle between pre- and post-balance training ranged from 0.45 degrees to 3.51 degrees. Minimum difference in club path angle was 0.88 degrees and maximum difference in club path angle was 1.91 degrees. Maximum change in range between pre- and post-balance training was 3.3 degrees for face angle and 0.4 degrees for club path. Minimum change in range was 0.24 degrees for face angle and o degrees for club path. Change in variance spanned from 0.40 to 3.02 for face angle and 0.25 to 0.01 for club path.

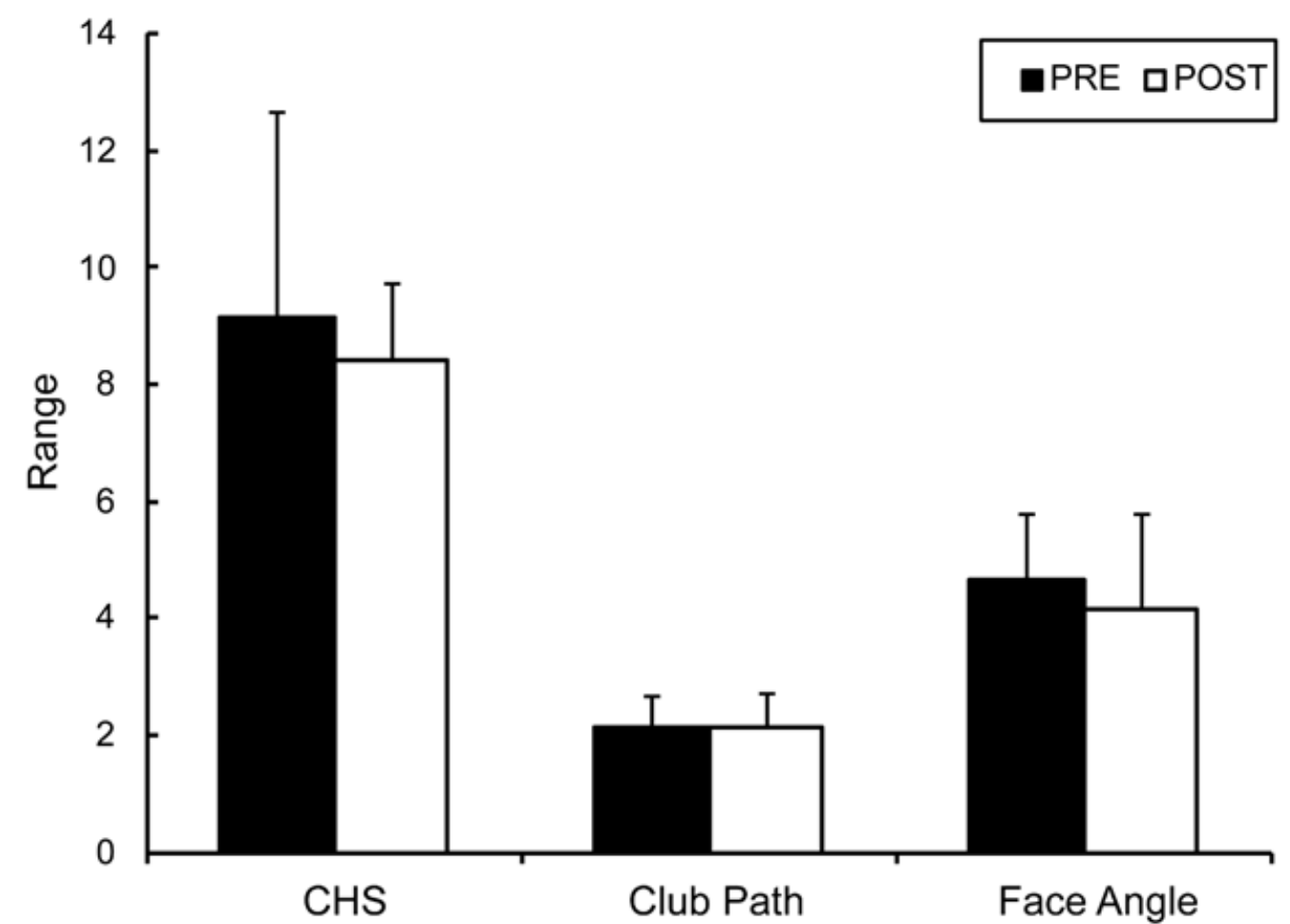

Figure-4. Range values for CHS, Club Path, and Face Angle were determined. Overall, the group trended toward decreased range for CHS and Face Angle. There were no statistically significant changes. Reported averages only include data for 7 subjects.

\subsubsection{Pelvis and Torso Angles}

The rotational angle of the pelvis and the torso were determined for the top of the swing, half down position, and impact position. No statistical difference in range was noted for any of the positions (pelvis top: $\mathrm{p}=0.52$, pelvis half down: $\mathrm{p}=0.70$, pelvis impact: $\mathrm{p}=0.18$, torso top: $\mathrm{p}=0.53$, torso half down $\mathrm{p}=0.55$, torso impact $\mathrm{p}=0.72$ ). However all but two positions trended toward decreased range following the balance training Figure 5. The average range increased for both the impact position for the pelvis and the top position for the torso. Individual range values decreased by a maximum of 4 degrees for the pelvis top and pelvis half down positions and by 1 degree 
for the pelvis impact position. Conversely, range values did increase for some participants with a maximum increase of 2 degrees for the pelvis top and pelvis half down positions and 3 degrees for the pelvis impact position. Individual range values for torso position decreased by a maximum of 2 degrees at the top position, 3 degrees at the half down position, and 4 degrees at the impact position. Individual range values increased by a maximum of 3 degrees at the top position, 4 degrees at the half down position, and 2 degrees at the impact position. Within the cohort of golfers, variance decreased by a maximum of $3.43,2.77$, and 3.21 at the top, half down, and impact positions for the torso, respectively. Variance increased by a maximum of 3.14, 1.29, and 3.34 at the top, half down, and impact positions for the torso, respectively.

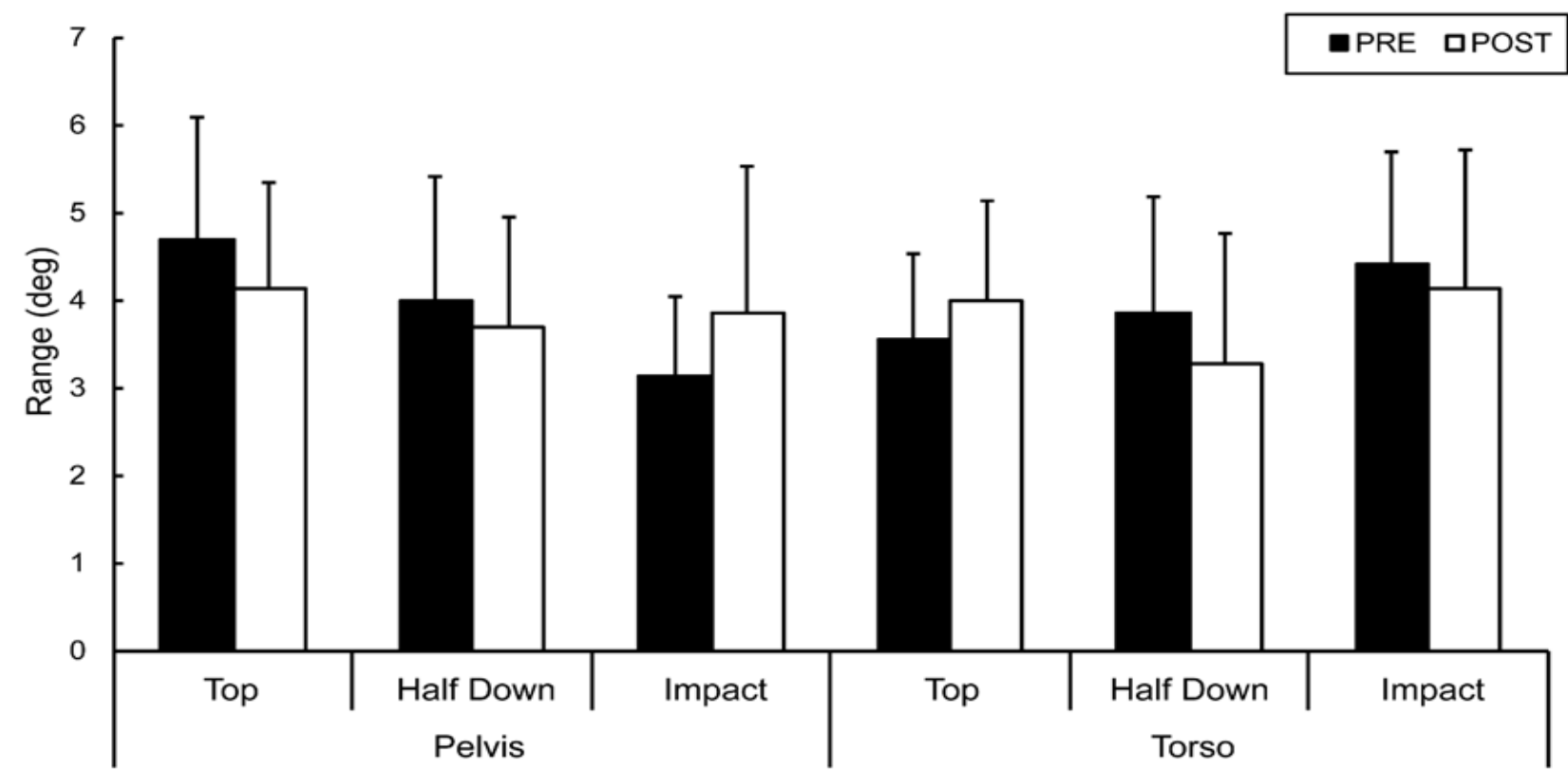

Figure-5. Range values for body segment positions were determined. There were no significant changes in range values for any of the body segment positions. Top is defined as the top of the swing, indicated by the transition period. Half down is defined as lead arm parallel to the ground.

\subsection{Swing Consistency Correlation to Balance Ability}

Overall, none of the selected metrics appear to have a significant linear correlation to postural stability as measured by the deviation in COP. Variance of the 8 swings for each metric was plotted against the $95 \%$ ellipse area for the COP deviation to determine trends.

Table-3. Pearson's correlation ( $\mathrm{r}$ ) and linear regression $\left(\mathrm{R}^{2}\right)$ values for pre- and post-test correlations between COP deviation and variance in swing kinematic variables. Correlation values were negligible for all kinematic variable. Only data from 7 subjects were included.

\begin{tabular}{c|c|c|c|c}
\hline & \multicolumn{2}{|c|}{ r } & \multicolumn{2}{c}{ R $^{2}$} \\
\hline & PRE & POST & PRE & POST \\
\hline CHS & 0.4924 & -0.1127 & 0.2425 & 0.0127 \\
\hline Club Path & 0.3065 & 0.6957 & 0.0940 & 0.4841 \\
\hline Face Angle & 0.0481 & 0.1020 & 0.5067 & 0.0104 \\
\hline Pelvis & & & & 0.0401 \\
\hline Top & 0.3012 & 0.2001 & 0.0907 & 0.1959 \\
\hline Half Down & 0.2871 & -0.4425 & 0.0824 & 0.0125 \\
\hline Impact & -0.0743 & 0.1116 & 0.0055 & 0.6244 \\
\hline Torso & & & & 0.1238 \\
\hline Top & 0.3517 & 0.7901 & 0.118 & 0.0006 \\
\hline Half Down & -0.3435 & 0.0252 & 0.0613 & 0.0606 \\
\hline
\end{tabular}


The single leg (left), eyes closed balance condition was selected for variance comparisons as a representative stance due to the significant improvements following the balance training noted in this stance condition. Table 3 lists the pre- and post-training linear regression $\left(\mathrm{R}^{2}\right)$ and Pearson's correlation ( $\mathrm{r}$ ) values for each metric. Determined values indicate no clear linear correlation between single leg, eyes closed postural stability and consistency in driver swings for pelvis and torso positions, club head speed, face angle, or club path. Our initial hypothesis that greater postural stability would correlate with increased swing consistency, as indicated by smaller variance for selected metrics, was not confirmed.

\section{DISCUSSION}

College athletics have become highly competitive with many elite level collegiate athletes aspiring to pursue careers in professional sports. Thus, many teams and athletes are seeking methods to improve performance in addition to standard skills practice, and the sport has seen a rise in the importance of strength and conditioning training. Additionally, consistency in swing movement is considered by many coaches to be a vital skill with direct influence on performance. The goal of the present study was to determine the effects of an 8-week golf specific balance training protocol on postural stability and swing kinematics, specifically consistency in swing kinematics. It was hypothesized that a greater level of postural stability and improvements in postural stability would correlate to better swing consistency and improvements in swing consistency, respectively. Subjects completed an 8-week balance training protocol and were assessed before and after training for postural stability and golf swing kinematics.

Postural stability showed significant improvement following the 8-week training protocol when assessed using the scoring methods for the Balance Error Scoring System (BESS). However, no statistically significant changes where noted in other measures of postural stability, such as deviation in the center of pressure, via the $95 \%$ ellipse area. Baseline measures of postural stability, pre-training, showed high levels of balance for all subjects. Elite golfers have been shown to have exceptional levels of balance when compared to lower proficiency golfers (Sell et al., 2007) and healthy non-golfers (Gao, Hui-Chan, \& Tsang, 2011) and the elite golfers in this study demonstrated this high level of postural stability at the pre-training assessment. With high levels of innate balance pre-training, large improvements in balance would be difficult to achieve. However, BES did improve for all golfers, indicating that there was less gross body movements noted visually. One potential explanation for the significant decrease in BES, but less significant decrease in 95\% ellipse area could be an increase in ankle mobility or compensation during difficult balance stances. Injury prevention is important for athletes of any sport and prior literature has indicated a relationship between balance ability and injury risk for a variety of sports (Hrysomallis, 2007; McGuine, Greene, Best, \& Leverson, 2000; Tropp, Ekstrand, \& Gillquist, 1984; Watson, 1999; Willems et al., 2005). Collegiate golfers are exposed to numerous activities that present a risk for injury, and while the results of this study indicate that balance improvements do not correlate directly to swing kinematics, all subjects did experience balance improvements that may help reduce the risk for injury during sport related activities.

For all swing parameters analyzed, consistency between repeated swing trials was statistically unaffected by the balance training. However, for CHS and face angle, the top and half down positions for the pelvis, and the half down and impact positions for the torso, the range decreased slightly. While the impact position is ultimately the deciding factor on the outcome of the shot, there is an agreed upon understanding that achieving the correct position at other time points in the swing is equally as important (Bradshaw et al., 2013). Decreasing the variability at these time points, including the top of the swing, could have positive effects on the overall consistency in repeated shot outcomes. Intercollegiate golfers have highly refined golf swings that are the result of much dedicated practice and coaching. Skilled golfers, of this nature, have been shown to have lower standard deviation values for inertial sensor measurements of swing kinematics when compared to beginner golfers (Lai, Hetchl, Wei, \& Ball, 2011). The cohort of intercollegiate, highly skilled golfers that was included in this study showed high levels of pretraining swing kinematic consistency as evidenced by the low range and variance values. Similar to balance 
improvements, statistical changes in swing consistency would be difficult to achieve with such a high level of initial swing consistency.

There were several limitations present in the current study that must be addressed and considered with the results. Firstly, the balance training exercises were completed in addition to all other standard team activities, which for collegiate golfers can include standard physical training, practice, course work, and competition travel. Thus, it is difficult to isolate the individual effects of the balance training independent from the other activities. Players were also expected to complete the balance training exercises on their own for days without scheduled workouts or when traveling. Additionally, the study featured no true control group because the focus of the study was on collegiate golfers similar to previous research (Doan et al., 2006). All subjects in the study completed the balance training exercises. The balance training was hypothesized to be beneficial to the players, so limiting which players received the balance training could have been considered a disadvantage. With all players competing for competition travel positions throughout the duration of the study, limiting which players received the training was not possible.

Many of the limitations present in the current study represent a realistic experience for all collegiate golfers. When evaluating the benefit of a certain training program, it is important to consider the outcomes of the training within the confines of an elite collegiate golf program. While this more realistic study design limits the definitive conclusions that can be drawn about the balance program itself, it does allow for a complete understanding of the results this type of training program would produce. Moreover, the balance training as well as the data collection throughout the 8-weeks showed no effects on the swing kinematics of the individual subjects. While consistency in swing kinematics did not improve after completion of the balance training, it did not decrease either indicating no harm from the study on the golfing ability of the individual participants.

\section{CONCLUSION}

Collegiate athletics has become highly competitive with athletes of all sports vying for the top finished in prestigious events. Thus, coaches and players have begun engaging in additional forms of training in combination with standard skills practice. With an abundance of training programs available, it is crucial for players and coaches to understand the potential benefits and make sure there are no negative effects of the training. Additionally, as previous studies have suggested, there are time constraints that must be considered when developing training and practice schedules for collegiate level athletes (Driggers \& Sato, 2018). Understanding when and if training is beneficial can help coaches and players better utilize the limited hours available as mandated by the NCAA. The results from this study indicate that participation in an 8-week golf specific balance training program improved overall balance for collegiate male golfers. Furthermore, there were no effects of the balance training on swing kinematics, indicating no negative effects of the training on swing performance. Overall, the initial hypothesis was not confirmed, and swing consistency was not affected by the balance training; however, pre-training consistency for all participants was initially very high which is to be expected for elite collegiate golfers. Strength, flexibility, and balance are all crucial skills for any elite golfer, and results from this study indicate that golf specific balance training does lead to improved balance but has no effect on swing consistency.

Funding: This study received no specific financial support.

Competing Interests: The authors declare that they have no competing interests.

Acknowledgement: The authors would like to acknowledge the Robert H. Brooks Sports

Science Institute for providing funding for the study. Additionally, the authors would like to thank Tommy Evans for his assistance with developing the balance intervention and the Clemson University Athletic Department for use of facilities.

\section{REFERENCES}

Bell, D. R., Guskiewicz, K. M., Clark, M. A., \& Padua, D. A. (2011). Systematic review of the balance error scoring system. Sports Health, 3(3), 287-295. Available at: https://dx.doi.org/10.1177/1941738111403122. 
Bradshaw, E. J., Keogh, J. W. L., Hume, P. A., Maulder, P. S., Nortje, J., \& Marnewick, M. (2013). The effect of biological movement variability on the performance of the golf swing in high- and low-handicapped players. Research Quarterly for Exercise and Sport, 8O(2), 185-196. Available at: https://dx.doi.org/10.1080/02701367.2009.10599552.

Cheetham, P. J., Martin, P. E., Mottram, R. E., \& Laurent, B. F. (2001). The importance of stretching the $\mathrm{x}$ factor in the golf downswing: The 'X-factor stretch.' In optimising performance in golf (pp. 192-199). Brisbane: Australian Academic Press.

Cheetham, P. J., Rose, G. A., Hinrichs, R. N., Neal, R. J., Mottram, R. E., Hurrion, P., D., \& Vint, P. F. (2008). Comparison of kinematic sequence parameters between amateur and professional golfers. Paper presented at the Poceedings of the World Scientific Congress of Golf, USA.

Doan, B. K., Newton, R. U., Kwon, Y., \& Kraemer, W. J. (2006). Effects of physical conditioning on intercollegiate golfer performance. The Journal of Strength and Conditioning Research, 20(1), 62-72. Available at: https://dx.doi.org/10.1519/R-17725.

Driggers, A. R., \& Sato, K. (2018). The effects of vertically oriented resistance training on golf drive performance in collegiate golfers. Sports Science $\Xi$ Coaching, 13(4), 598-606. Available at: https://doi.org/10.1177/174795411 17743374.

Fletcher, I. M., \& Hartwell, M. (2004). Effect of an 8-week combined weights and plyometrics training program on golf drive performance. Journal of Strength and Conditioning Research, 18(1), 59-62. Available at: https://dx.doi.org/10.1519/1533-4287(2004)0 18<0059:eoawcw>2.0.co;2.

Gao, K. L., Hui-Chan, C. W., \& Tsang, W. W. (2011). Golfers have better balance control and confidence than healthy controls. European Journal of Applied Physiology, 111 (11), 2805-2812. Available at: https://doi.org/10.1007/s0042 1-011-1910-7.

Goldie, P. A., Bach, T., \& Evans, O. (1989). Force platform measures for evaluating postural control: Reliability and validity. Archives of Physical medicine and Rehabilitation, 7O(7), 510-517.

Gordon, B. A., Moir, G. L., Davis, S. E., Witmer, C. A., \& Cummings, D. M. (2009). An investigation into the relationship of flexibility, power, and strength to club head speed in male golfers. Journal of Strength and Conditioning Research, 23(5), 1606-1610. Available at: https://dx.doi.org/10.1519/JSC.0b013e3181a3c39d.

Gulgin, H. R., Schulte, B. C., \& Crawley, A. A. (2014). Correlation of titleist performance institute level 1 movement screens and golf swing faults. The Journal of Strength \& Conditioning Research, 28(2), 534-539. Available at: https://doi.org/10.1519/jsc.obo13e31829b2ac4.

Hrysomallis, C. (2007). Relationship between balance ability, training and sports injury risk. Sports Medicine, 37(6), 547-556. Available at: https://doi.org/10.2165/00007256-200737060-00007.

Lai, D. T. H., Hetchl, M., Wei, X., \& Ball, K. (2011). On the difference in swing arm kinematics between low handicap golfers and non-golfers using wireless inertial sensors. Procedia Engineering, 13, 219-225. Available at: https://doi.org/10.1016/j.proeng.2011.05.076.

Langdown, B. L., Bridge, M., \& Li, F.-L. (2012). Movement variability in the golf swing. Sports Biomechanics, $11(2), 273-287$. Available at: https://dx.doi.org/10.1080/14763141.2011.650187.

Lephart, S. M., Smoliga, J. M., Myer, J. B., Sell, T. C., \& Tsai, Y.-S. (2007). An eight-week golf-specific exercise program improves physical characteristics, swing mechanics, and golf performance in recreational golfers. . Journal of Strength and Conditioning Research 21(3), 860-869. Available at: https://dx.doi.org/10.1519/R-20606.1.

Marshall, K. J., \& Llewellyn, T. L. (2017). Effects of flexibility and balance on driving distance and club head speed in collegiate golfers. International Journal of Exercise Science, 10(7), 954-963.

McGuine, T. A., Greene, J. J., Best, T., \& Leverson, G. (2000). Balance as a predictor of ankle injuries in high school basketball players. Clinical Journal of Sport Medicine, 10(4), 239-244. Available at: https://dx.doi.org/10.1097/00042752$200010000-00003$.

Oranchuk, D. J., Mannerberg, J. M., Robinson, T. L., \& Nelson, M. C. (2020). Eight weeks of strength and power training improves club head speed in collegiate golfers. The Journal of Strength \& Conditioning Research, 34(8), 2205-2213. Available at: https://dx.doi.org/10.1519/JSC.0000000000002505. 
Parker, J., Lagerhem, C., Hellström, J., \& Olsson, M. C. (2017). Effects of nine weeks isokinetic training on power, golf kinematics, and driver performance in pre-elite golfers. BMC Sports Science, Medicine and Rehabilitation, 9(1), 1-12. Available at: https://dx.doi.org/10.1186/s13102-017-0086-9.

Qualisys, A. (2019). Golf module user manual. Gothenburg, Sweden: Qualisys AB.

Riemann, B. L., Guskiewicz, K. M., \& Shields, E. W. (1999). Relationship between clinical and forceplate measures of postural stability. Journal of Sport Rehabilitation, 8(2), 71-82.

Sell, T. C., Tsai, Y.-S., Smoliga, J. M., Myers, J. B., \& Lephart, S. M. (2007). Strength, flexibility, and balance characteristics of highly proficient golfers. The Journal of Strength \& Conditioning Research, 21(4), 1166-1171.

Tropp, H., Ekstrand, J., \& Gillquist, J. (1984). Stabilometry in functional instability of the ankle and its value in predicting injury. Medicine $\mathbb{E}^{2}$ Science in Sports $\mathbb{E}^{2}$ Exercise, 16(1), 64-66.

Watson, A. W. S. (1999). Ankle sprains in players of the field-games Gaelic football and hurling. Journal of Sports Medicine and Physical Fitness, 39(1), 66-70.

Weston, M., Coleman, N. J., \& Spears, I. R. (2013). The effect of isolated core training on selected measures of golf swing performance. Medicine $\Xi^{\circ}$ Science in Sports $\Xi^{\circ}$ Exercise, 45(12), 2292-2297. Available at: https://dx.doi.org/10.1249/MSS.ob013e31829bc7af.

Willems, T. M., Witvrouw, E., Delbaere, K., Mahieu, N., De Bourdeaudhuij, L., \& De Clercq, D. (2005). Intrinsic risk factors for inversion ankle sprains in male subjects: A prospective study. The American Journal of Sports Medicine, 33(3), 415-423. Available at: https://dx.doi.org/10.1177/0363546504268137. 